\title{
Revisión Sistemática: La profilaxis precoz con heparina de bajo peso podría ser más protectora de trombosis venosa profunda en la artroplastia de cadera
}

Timing of initial administration of low-molecular-weight heparin prophylaxis against deep vein thrombosis in patients following elective hip arthroplasty. Hull RD, Pineo GF, Stein PD y cols. Arch Int Med 2001; 161: 1952-1960

\section{Objetivo}

Determinar si la profilaxis con heparinas de bajo peso molecular (HBPM) en pacientes con reemplazo de cadera, es mejor cuando la droga se administra cercanamente $(<12 \mathrm{hs})$ o alejadamente $(>12 \mathrm{hs})$ de la cirugía, con respecto a la anticoagulación oral.

Lugar

Revisores canadienses, noruegos, holandeses y de Estados Unidos. Diseño

Revisión sistemática de estudios publicados que comparaban HBPM con anticoagulantes orales en pacientes con reemplazo de cadera y cuyo resultado primario fuera trombosis venosa profunda (TVP) diagnosticada por flebografía contrastada bilateral.

Fuente y selección de estudios

Se realizó una búsqueda exhaustiva en MEDLINE, a partir de citas de los mismos estudios encontrados, de conferencias y contacto con los autores y la industria farmacéutica.Criterios de inclusión:1-que enrolaran pacientes con reemplazo de cadera electivo; 2- que fueran asignados aleatoriamente al tratamiento;3- que investigaran efectividad y seguridad de la HBPM administrada una o dos veces al día; 4- que documenten objetivamente la presencia de TVP con flebografía bilateral ascendente;5- que documenten objetivamente la presencia de complicaciones hemorrágicas.

\section{Medición de resultados principales}

Se obtuvo información sobre la frecuencia de TVP y complicaciones hemorrágicas en ambas ramas de tratamiento de los estudios.

Resultados principales

Cuatro estudios cumplieron los criterios de inclusión, dos de los cuales fueron doble ciego.En uno de ellos se administró heparina alejadamente (18-24 hs postcirugía); en otro alejadamente precirugía, y en los dos restantes cercanamente a la cirugía (pre y postoperatoria).Las flebografías exitosas (de buena calidad) estuvieron en el orden del $75 \%$.Los trabajos utilizaron diferentes HBPM (tinza, nadro y dalteparine), diferentes anticoagulantes (acenocumarol o wafarina), y distintas dosis iniciales (usual o mitad de la usual) y momento de administración (18-24 hs.en la tarde del día previo a la cirugía, 2 hs pre, 4-6 hs post) y el tiempo entre la cirugía y la flebografía (5.7 a 10 días).La HBPM administrada cercana al procedimiento quirúrgico mostró una reducción relativa del riesgo entre el 43 y el $55 \%$ con respecto a anticoagulantes orales;mientras que alejadamente del procedimiento no mostró diferencias con los ACO.

\section{Conclusiones}

En pacientes con reemplazo de cadera programado, la profilaxis de trombosis venosa profunda con heparinas de bajo peso molecular podría ser mejor si la misma se administra en el perioperatorio cercano.

\section{Comentario}

Cuando surgieron los meta-análisis y revisiones sistemáticas en el ámbito de la investigación científica, mucho se escribió sobre la cantidad de avances que se hubieran hecho en medicina, con solo "amontonar y ordenar" la evidencia dispersa en muchos ensayos clínicos. Es el conocido caso de la trombolisis coronaria'.

Una revisión sistemática intenta reunir toda la mejor evidencia disponible sobre un determinado interrogante clínico y de esta manera aumentar el poder para contestar esa pregunta, ampliando la diversidad de poblaciones en las cuales se implementó un determinado tratamiento.

En este estudio, la pregunta científica propuesta por los autores: " comparado con el uso de ACO, cuándo es mejor iniciar la profilaxis anticoagulante con HBPM en pacientes que serán sometidos a un reemplazo de cadera?", fue el desencadenante de uno solo de los trabajos que pudieron recabarse (mostró mejores resultados con HBPM pre o postquirúrgica, con respecto a wafarina). Los autores se disponen a contestar su pregunta con el agregado de más información: algunos de los pacientes (los que se operan de cadera) de una de las ramas de tratamiento (la de los que reciben HBPM) de estudios que utilizaron HBPM en comparación con anticoagulación oral.Ningún artículo se cuestionaba específicamente si era mejor iniciar la profilaxis cerca o lejos del procedimiento. Por lo tanto, las conclusiones que podamos sacar de este estudio, deben ser miradas con el mismo prisma que se analizan las conclusiones de los análisis de subgrupos en ensayos clínicos de envergadura: "este hallazgo puede ser real pero no era lo que se estaba buscando". Los hallazgos pueden explicarse porque en uno de los estudios "alejados" no hubo diferencias debido a que los que recibieron ACO tuvieron baja incidencia de TVP, mientras que en el otro "alejado" fue alta la incidencia en el grupo HBPM.Estos a su vez son los dos estudios más antiguos.
Llama la atención que los autores de los cuatro trabajos que cumplieron los criterios de inclusión predefinidos son, al mismo tiempo, los autores de esta revisión sistemática.

La decisión del momento de administrar la profilaxis con heparina está lejos de ser dilucidada con este estudio. Mientras tanto, en el ámbito donde se define esta problemática: con unos especialistas que sufren los sangrados intraoperatorios, y otros especialistas que sufren las complicaciones trombóticas, hacen de esta pregunta un interrogante que amerita prontas respuestas de ensayos clínicos específicamente diseñados. Por otro lado, aunque no tengamos esta respuesta siguen surgiendo nuevas drogas (inhibidores del factor $X$ activado $^{2}$ que se postulan para desplazar a las que aún no sabemos usar en su máximo provecho.La evidencia siempre llega más tarde que la propaganda médica.

Otro dato interesante, para quien se enfrenta diariamente al paciente, es la alta incidencia de TVP reportadas en estos estudios (hasta del $25 \%$ de los pacientes que recibieron profilaxis oral y $13 \%$ en los que recibieron HBPM).La definición flebográfica de TVP utilizada es quizás mucho más contundente que la definición que podemos usar en la práctica diaria.

Por último, los criterios que se utilizan en este tipo de revisiones para definir la calidad de los estudios que se incluyen son tan variados, que un estudio interesante, precisamente evaluando 25 escalas diferentes en ensayos clínicos con HBPM demuestra que según el puntaje que se use, las conclusiones que se obtienen pueden ser disímiles y hasta opuestas ${ }^{3}$.

Conclusión del comentario: A pesar que las heparinas de bajo peso molecular podrían ser mejor que la anticoagulación administrada en el perioperatorio cercano, habrá que esperar nuevos ensayos clínicos para respoder esta pregunta en forma definitiva.

\section{Dr. Juan P. Roubicek [ Servicio de Clínica Médica. Instituto Médico Bolívar de Mar del Plata ]}

\section{Referencias}

1. Lau J, Antman EM, Jimenez-Silva J, Kupelnick B, Mosteller F, Chalmers TC. Cumulative meta-analysis of therapeutic trials for myocardial infarction. N Engl J Med.1992 Jul 23:327(4):248-54. 2. Turpie AG. Pentasaccharide Org31540/SR90107A clinical trials update:lessons for practice.Am Heart J.2001 Aug:142(2 Suppl):S9-15.

3. Juni P, Witschi A, Bloch R y col.The Hazards of scoring quality of clinical trials for meta-analysis.JAMA 1999;282:1054-1060. 